J. Phys. IV France 130 (2005) 203-207

(C) EDP Sciences, Les Ulis

DOI: $10.1051 / \mathrm{jp} 4: 2005130014$

\title{
L'exemple de la détermination de la structure cristallographique de la nucléocapside du bluetongue virus
}

\author{
P. Gouet \\ Laboratoire de BioCristallographie-IBCP, UMR 5086 CNRS-UCBL, 7 passage du Vercors, \\ 69367 Lyon Cedex 07, France
}

\begin{abstract}
Résumé. Les virus sont responsables de plus de la moitié des maladies infectieuses développées chez l'homme. Ils sont caractérisés par un génome de petite taille, enfermé dans une capside résultant de l'assemblage de sous-unités identiques. Cette encapsidation préserve le génome viral contre les dispositifs de défense de l'organisme infecté. La compréhension des mécanismes d'assemblage peut être décisive dans l'élaboration de nouveaux médicaments.

La structure 3D de la nucléocapside du bluetongue virus a été résolue par cristallographie aux rayons X. Elle présente un assemblage icosaédrique remarquable, avec une capside de diamètre 700 Å formée de deux protéines différentes appelées VP3 et VP7. Les cartes de densité électronique montrent que le génome viral se structure autour de polymérases, disposées selon les axes de symétrie d'ordre 5 de l'icosaèdre.
\end{abstract}

\section{INTRODUCTION}

La résolution de la structure d'un virus à l'échelle atomique peut permettre de mieux comprendre au niveau moléculaire son mécanisme d'infection, de réplication et d'assemblage. Une finalité de ces recherches structurales est d'aider à la conception de médicaments susceptibles de bloquer ces mécanismes viraux. Elles peuvent également participer à la création de vaccins synthétiques, possédant les caractéristiques du virus et susceptibles de déclencher une réponse immunitaire appropriée. La plupart des virus présentent une capside sphérique, résultant de l'organisation de protéines virales suivant une symétrie icosaédrique. Un icosaèdre comprend 60 éléments identiques, reliés par des opérateurs de symétrie de rotation 2, 3 et 5. Chaque élément peut être constitué de plusieurs chaînes polypeptidiques différentes ou identiques en séquence. Ce nombre de chaînes, également appelé nombre de triangulation $\mathrm{T}$, obéit à une formule mathématique $T=h^{2}+h k+k^{2}$ avec $\mathrm{h}$ et $\mathrm{k}$ entiers. Ainsi $\mathrm{T}=1$ pour $\mathrm{h}=1, \mathrm{k}=0$ et l'icosaèdre comprend 60 chaînes; $\mathrm{T}=3$ pour $\mathrm{h}=1, \mathrm{k}=1$ et l'icosaèdre comprend $3 \times 60=180$ chaînes etc. Toutes les chaînes ne voient pas exactement le même environnement lorsque $\mathrm{T}$ est supérieur à 1 . Elles s'associent alors selon une règle de quasi-équivalence [1]. En conséquence, une capside icosaédrique formée de 180 chaînes est assemblée selon une base de trois chaînes, dont la structure est quasiment identique.

\section{LES ORBIVIRUS}

Le travail effectué dans le groupe du professeur David Stuart au Laboratory of Molecular Biophysics d'Oxford, Angleterre, a permis de déterminer en 1998 la structure de la nucléocapside du virus de la fièvre catharrale maligne du mouton (en anglais bluetongue virus, BTV) [4]. Ce virus appartient au genre orbivirus de la famille des Reoviridae, qui compte aussi african horsesickness virus (AHSV) et epizootic-hemorrhagic disease virus (EHDV). Les orbivirus sont transmis par des insectes (Culicoïdes) et infectent le bétail des régions tropicales et subtropicales, causant de graves dégâts économiques. Ils ont un diamètre de $800 \AA$ Å et un poids moléculaire de 100 millions de Dalton. La détermination de telles structures à l'échelle atomique représente un véritable défi cristallographique.

Les orbivirus sont constitués d'une double enveloppe protéique de symétrie icosaédrique enfermant un génome de $10 \mathrm{ARN}$ double brin (ARNdb). Chaque ARNdb code pour une protéine virale. L'enveloppe 


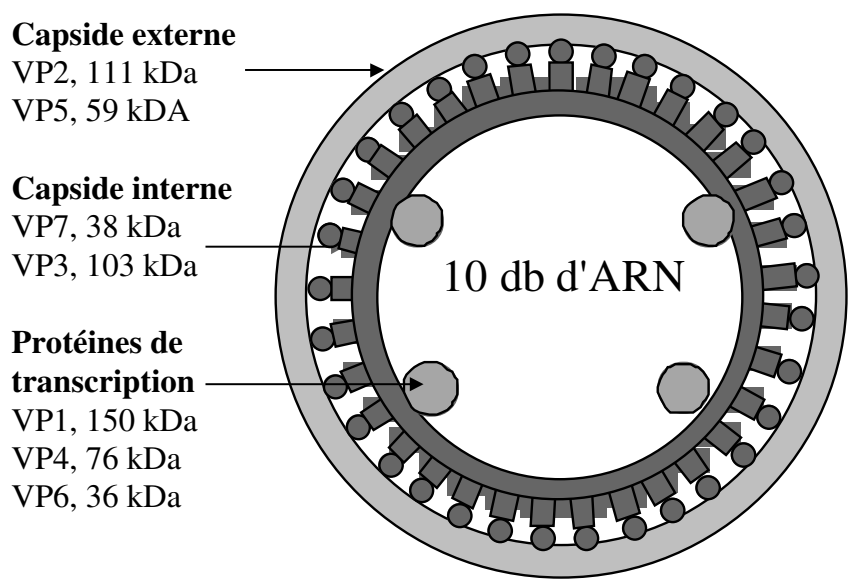

Figure 1. Schéma de coupe d'un orbivirus.

externe du virus est formée d'un arrangement de protéines structurales appelées VP2 et VP5 (figure 1). L'enveloppe interne de diamètre 700 Å est constituée des protéines VP3 et VP7. Le tout enferme l'ARNdb génomique et un complexe polymérase constitué des protéines VP1, 4 et 6 . Les trois dernières protéines codées, NS1, 2 et 3, ne sont pas structurales et sont absentes de la particule virale.

\section{RÉSOLUTION DE LA STRUCTURE ATOMIQUE DE LA NUCLÉOCAPSIDE DE BTV}

\subsection{Résolution de la structure cristallographique de VP7}

La résolution cristallographique d'une structure virale se fait généralement pas à pas. La structure d'une protéine de capside est obtenue en premier. Cette structure sera ensuite placée dans des cartes de reconstruction de microscopie électronique, afin de générer un modèle de la capside pour l'ensemble des protéines de même type. Ce modèle pourra alors être utilisé pour résoudre le problème des phases par la méthode du remplacement moléculaire, après avoir enregistré des données rayons $\mathrm{X}$ sur des cristaux de virus.

Ainsi, la protéine de capside VP7 du BTV a été isolée, cristallisée et sa structure a été résolue par la méthode des dérivés lourds à 2,6 Å de résolution [3]. Il s'agit d'un trimère de 3x349 résidus, constitué d'un domaine inférieur en hélices $\alpha$ et d'un domaine supérieur en brins $\beta$ (figure 2). Le domaine inférieur est posé sur la couche de VP3 dans le virus entier, tandis que le domaine supérieur supporte la capside externe.

La structure cristallographique de VP7 a ensuite été positionnée dans des cartes de reconstruction de la nucléocapside du BTV, obtenues par microscopie électronique à une résolution de $22 \AA$ [5]. Ces cartes montraient que la capside interne était formée de 780 monomères de VP7, arrangés selon un réseau quasi équivalent T=13levo. Un modèle 3D pour l'arrangement de VP7 dans la nucléocapside a ainsi été construit (figure 3).

\subsection{La résolution de la nucléocapside du BTV}

Les tentatives de cristallisation du BTV entiers ont été infructueuses. Par contre, des cristaux des nucléocapsides des sérotypes 1 et 10 du BTV ont été obtenus. Le sérotype 1 est isolé en Afrique du Sud, le 10 des USA. Les nucléocapsides des deux sérotypes sont fortement homologues (plus de $90 \%$ d'identité de séquence protéique) mais elles cristallisent dans des groupes d'espace différents : P $2{ }_{1} 2{ }_{1} 2$ pour BTV-1 avec pour paramètres de maille $\mathrm{a}=796 \AA$, $\mathrm{b}=825 \AA$, $\mathrm{c}=756 \AA ̊ \mathrm{P}$; $4{ }_{1} 2{ }_{1} 2$ pour BTV-10 avec 


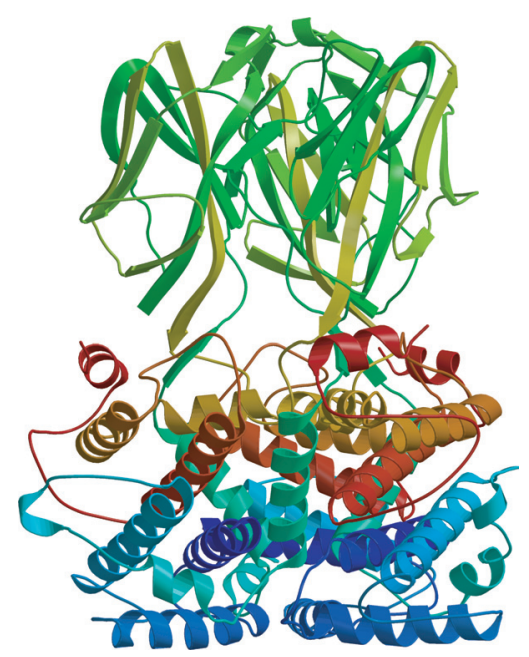

Figure 2. Tracé en mode ruban du trimère VP7 du BTV.

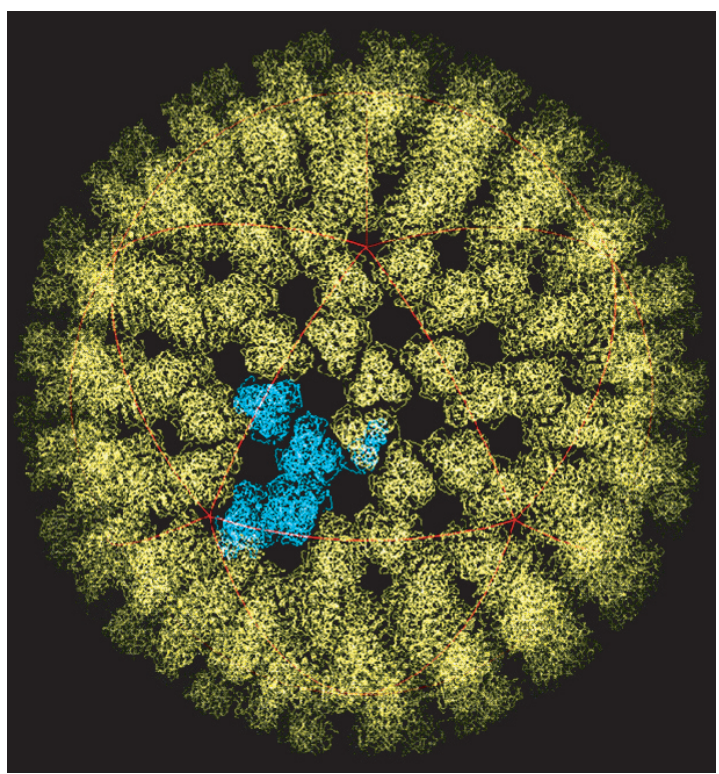

Figure 3. Arrangement de la protéine VP7 sur la nucléocapside du BTV. 13 monomères de VP7 sont assemblés sur l'unité asymétrique de l'icosaèdre (en bleu). 13x60 monomères constituent la nucléocapside. Les axes de symétrie 5 de l'icosaèdre sont reliés, délimitant 20 faces triangulaires.

$\mathrm{a}=\mathrm{b}=1115 \AA \mathrm{c}, \mathrm{c}=1584 \AA$. Ces cristaux renferment le génome du virus et sont potentiellement infectieux. Aussi, leur cristallisation est-elle réalisée par l'équipe de Peter Mertens dans un environnement de niveau de sécurité P3, à l'Institut for Animal Health (IAH), Pirbright en Angleterre. Les cristaux sont montés sur place dans des capillaires de verre scellés et amenés sur les sites de rayonnement synchrotron (SRS Daresbury, Angleterre ou ESRF Grenoble, France) sous la responsabilité d'agents de sécurité. Les cristaux en capillaire sont refroidis à $4^{\circ} \mathrm{C}$ pendant l'enregistrement. Ils sont fragiles et seulement 1-6 images de diffraction sont mesurées par cristal. Un jeu complet avec une bonne résolution (inférieure à $6 \AA$ A nécessite l'emploi de centaines de cristaux. Les données synchrotron ont ainsi été enregistrées au cours 
de nombreux voyages à Daresbury et à Grenoble. Les cristaux du BTV-1 peuvent diffracter jusqu'à une résolution de 3,5 $\AA$, ceux du BTV-10 jusqu'à $6,5 \AA$.

La structure cristallographique de la nucléocapside du BTV-1 a été résolue en premier par la méthode du remplacement moléculaire. La reconstruction de la nucléocapside avec VP7 a été prise comme modèle (figure 3) pour résoudre le problème des phases. De nombreux cycles d'affinement ont permis d'homogénéiser les cartes de densité électronique en utilisant les opérateurs de symétrie non-cristallographique. La densité électronique correspondant à la structure de la deuxième protéine de capside, VP3, a ainsi pu être observée et la structure de cette protéine a été construite. Après affinement cristallographique, il est apparu que VP3 était constituée de trois domaines susceptibles de pivoter et qu'elle s'arrangeait sur la nucléocapside suivant un réseau pseudo $\mathrm{T}=2$ [4]. Cette flexibilité entre domaines est nécessaire pour l' assemblement de VP3 suivant ce réseau qui ne respecte pas le principe de la quasi-équivalence ( $\mathrm{T}=2$ n'est pas un nombre de triangulation permis). La structure de BTV-1 comprenant VP3 et VP7 a alors servi de modèle pour résoudre celle de BTV-10 par remplacement moléculaire. Des cartes de densité très semblables ont été obtenues.

\subsection{La structuration du génome viral de BTV}

Les formes des structures des complexes polymérases de même que celles de l'ARNdb viral sont apparues à l'intérieur des capsides de BTV-1 et BTV-10, après calcul de cartes de densité électronique à basse résolution. Ces complexes macromoléculaires ne sont pas présents en un multiple de 60 dans le virus et ne peuvent obéir à la symétrie icosaédrique Leurs structures 3D ne pourront donc pas être résolues à l'échelle atomique, à partir de données rayons $\mathrm{X}$ enregistrées sur des cristaux de virus. Ces macromolécules sont toutefois suffisamment ordonnées dans le virus, pour être visible dans des cartes de densité électronique convoluées et moyennées à une résolution de 6,5 ̊. Un modèle 3D de l'ARNdb dans le virus a ainsi pu être construit (figure 4) [2].

Ce modèle représente $80 \%$ des 19000 paires de bases du génome viral. Il montre que chaque segment d'ARNdb est susceptible de s'enrouler autour d'un complexe polymérase, le long des axes icosaédriques

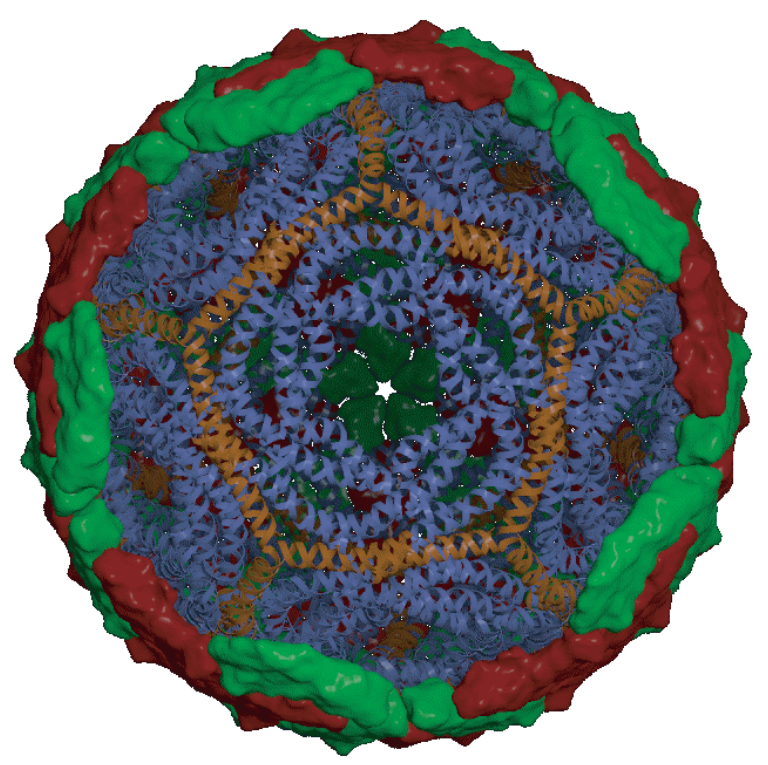

Figure 4. Modèle du repliement de l'ARN double brin sous la couche VP3 de la capside interne. L'ARNdb s'arrange en spirale autour des axes 5 icosaédriques. 
de symétrie 5. Un segment d'ARNdb pourrait ainsi s'étager en spirale sur plusieurs niveaux. Cette architecture est vraisemblablement commune à l'ensemble des Reoviridae. Elle est susceptible de leur donner une grande efficacité, lors de la synthèse de l'ARNm viral faisant suite à l'entrée du virus dans la cellule. Cet ARNm est utilisé par la machinerie cellulaire de l'hôte afin de produire de nouvelles particules virales et une telle efficience participe à la vitesse de propagation du virus dans l'organisme.

\section{CONCLUSION}

L'ensemble de ces données a permis d'affiner le scénario proposé pour la formation de la nucléocapside du BTV : des décamères de VP3 se formeraient en premier, 12 décamères s'assemblant pour constituer la première capside virale. Les complexes polymérases seraient alors intégrés à l'intérieur de cette protocapside ; puis 10 segments d'ARN simple brin correspondant au génome viral seraient isolés à la surface de cette proto-capside et incorporés ; durant leur incorporation, la synthèse de l'ARNdb serait réalisée par les complexes polymérases déjà encapsidés ; enfin, 260 trimères de VP7 viendraient s'assembler à la surface afin de constituer la nucléocapside finale. L'élaboration de composés bloquant ce mécanisme d'assemblage et/ou la sortie de l'ARNm viral le long des axes de symétrie 5 portant les complexes polymérases pourrait être envisagée.

\section{Références}

[1] Caspar D.L.D. and Klug A., Quant. Biol. 27 (1962) 1-24.

[2] Gouet P., Diprose J.M., Grimes J.M., Malby R., Burroughs J.N., Zientara S., Stuart D.I. and Mertens P.P.C. Cell 97 (1999) 481-490.

[3] Grimes J., Basak A.K., Roy P. and Stuart D., Nature 373 (1995) 167-170.

[4] Grimes J.M., Burroughs J.N., Gouet P., Diprose J.M., Malby R., Zientara S., Mertens P.P. and Stuart D.I., Nature 395 (1998) 470-478.

[5] Grimes J.M., Jakana J., Ghosh M., Basak A.K., Chiu W., Stuart D.I. and Prasad B.V., Structure 5 (1997) 885-893. 